\title{
Conhecimento de pacientes em hemodiálise quanto ao autocuidado com cateter venoso central
}

\author{
Knowledge of patients in hemodialysis as to self-care with central venous cateter \\ Conocimiento de pacientes en hemodiálisis en cuanto al autocuidado con catéter venoso \\ central
}

\begin{abstract}
Evelym Cristina da Silva Coelho ${ }^{1 *}$, Helloyza Halana Fernanda Aquino Pompeu', Ilma Pastana Ferreira $^{1}$, Auriceli Da Silva Souza ${ }^{2}$, Francinea de Nazaré Ferreira de Castilho ${ }^{1}$, Vera Lúcia Cecim dos Santos ${ }^{1}$, Adilson Mendes De Figueiredo Junior ${ }^{1}$, Jaqueline Vieira Guimarães ${ }^{1}$
\end{abstract}

\section{RESUMO}

Objetivo: Analisar o conhecimento de pacientes em hemodiálise sobre o autocuidado com o cateter venoso central. Método: Estudo descritivo de abordagem qualitativa realizado com 9 pacientes matriculados no Serviço de Terapia Renal Substitutiva da Fundação Hospital de Clínicas Gaspar Vianna, no município de Belém do Pará. Utilizou-se um instrumento para a coleta dos dados que posteriormente foi analisado segundo o modelo proposto por Bardin. Resultados: Após as a análise e interpretação dos dados coletados dos 09 pacientes, os quais compuseram a amostra do estudo, emergiram 3 categorias: Conhecimento e significância da hemodiálise na vida dos pacientes renais crônicos; $O$ autocuidado e o cotidiano do paciente renal crônico em hemodiálise; A autoestima e autoimagem do paciente renal crônico em hemodiálise. Percebeu-se que de maneira geral todos os pacientes possuem carência de conhecimento acerca de seu tratamento e sobre o autocuidado necessário à manutenção correta de seu cateter. Conclusão: Destaca-se a importância do enfermeiro no repasse de orientações para a promoção do autocuidado dos pacientes renais crônicos e, consequentemente, melhor qualidade de vida. Sugerimos a construção de tecnologias educativas para uso com estes pacientes, além da implementação da Sistematização da Assistência de Enfermagem, a qual poderá identificar as deficiências no autocuidado e implementar as intervenções de enfermagem minimizando tal problemática.

Palavra chave: Hemodiálise, Insuficiência Renal Crônica, Cuidado de enfermagem, Autocuidado.

\begin{abstract}
Objective: To analyze the knowledge of patients on hemodialysis on self-care with the central venous catheter. Method: Descriptive study of a qualitative approach performed with 9 patients enrolled in the Substitute Renal Therapy Service of the Hospital de Clínicas Gaspar Vianna Foundation, in the city of Belém do Pará. An instrument was used to collect data that was later analyzed according to the model proposed by Bardin. Results: After the analysis and interpretation of the data collected from the 9 patients, who composed the study sample, 3 categories emerged: Knowledge and significance of hemodialysis in the life of chronic renal patients; Self-care and daily life of the chronic renal patient on hemodialysis; The self-esteem and self-image of the chronic renal patient on hemodialysis. It was noticed that in general all patients have a lack of knowledge about their treatment and about the self-care necessary for the correct maintenance of their catheter. Conclusion: It is important to emphasize the importance of the nurse in passing on guidelines for the promotion of self-care of chronic renal patients and, consequently, a better quality of life. We suggest the construction of educational technologies for use with these patients, in addition to the implementation of Nursing Care Systematization, which can identify deficiencies in self-care and implement nursing interventions, minimizing such problems
\end{abstract}

Keywords: Hemodialysis, Chronic Renal Insufficiency, Nursing Care, Self-Care.

\footnotetext{
1Universidade do Estado do Pará (UEPA), Belém-Pará. Pará. *E-mail: evelym titina@hotmail.com 2Universidade Federal de São Paulo (UNIFESP).
}

SUBMETIDO EM: 9/2018

ACEITO EM: 10/2018

PUBLICADO EM: 12/2018 


\section{RESUMEN}

Objetivo: Analizar el conocimiento de pacientes en hemodiálisis sobre el autocuidado con el catéter venoso central. Método: Estudio descriptivo de abordaje cualitativo realizado con 9 pacientes matriculados en el Servicio de Terapia Renal Substitutiva de la Fundación Hospital de Clínicas Gaspar Vianna, en el municipio de Belém do Pará. Se utilizó un instrumento para la recolección de los datos que posteriormente fue analizado según el modelo propuesto por Bardin. Resultados: Después del análisis e interpretación de los datos recolectados de los 09 pacientes, los cuales compusieron la muestra del estudio, surgieron 3 categorías: Conocimiento y significancia de la hemodiálisis en la vida de los pacientes renales crónicos; EI autocuidado y el cotidiano del paciente renal crónico en hemodiálisis; La autoestima y autoimagen del paciente renal crónico en hemodiálisis. Se percibió que de manera general todos los pacientes poseen carencia de conocimiento acerca de su tratamiento y sobre el autocuidado necesario para el mantenimiento correcto de su catéter. Conclusión: Se destaca la importancia del enfermero en el traspaso de orientaciones para la promoción del autocuidado de los pacientes renales crónicos y, consecuentemente, mejor calidad de vida. Sugerimos la construcción de tecnologías educativas para uso con estos pacientes, además de la implementación de la Sistematización de la Asistencia de Enfermería, la cual podrá identificar las deficiencias en el autocuidado e implementar las intervenciones de enfermería minimizando tal problemática.

Palabra clave: Hemodiálisis, Insuficiencia Renal Crónica, Cuidado de enfermería, Autocuidado.

\section{INTRODUÇÃO}

De acordo com Salimena et al. (2016), a Doença Renal Crônica (DRC) é definida pela diminuição progressiva do ritmo da filtração glomerular, causando desiquilíbrio no organismo, já que os rins são responsáveis por esta manutenção. Havendo, também o comprometimento das funções regulatórias, excretórias e endócrinas envolvendo os demais órgãos do corpo.

Segundo estimativas da Sociedade Brasileira de Nefrologia (2016), em 2015 mais de 1,5 milhões de pessoas estiveram em Terapia Renal Substitutiva (TRS) (Hemodiálise, Diálise peritoneal, Transplantes renal), sendo 113 mil em diálise apenas no Brasil. Com aumento mundial, da taxa de doença renal em $10 \%$ ao ano. Assim a DRC configura-se como um dos agravos e que alcança altos índices de incidência no Brasil e no mundo. Um estudo da National Health and Nutrition Examination Survey (NHANES), que utilizou como amostra indivíduos com 20 anos ou mais, constatou que aproximadamente $13 \%$ da população adulta dos Estados Unidos tem DRC estágios 1 a 4. (BASTOS e KIRSZTAJN, 2011).

Segundo Guimarães et al. (2017), dentre as Terapias Renais Substitutivas (TRS) o tratamento mais utilizado no Brasil e no mundo é a hemodiálise (HD), para esta modalidade tem-se o material tecnológico necessário, os insumos, os profissionais especializados, afim de se obter o acesso vascular (AV) devido no paciente, dentre estes acessos os mais utilizados são, fístula arteriovenosa (FAV), cateter de curta permanência (cateter duplo lúmen) e de longa permanência (permicath).

Zica (2016) reitera que o AV é decisivo no tratamento de pacientes em HD, pois a manutenção deste acesso está diretamente ligada à qualidade de vida deste individuo e sua sobrevivência. As complicações mais prevalentes no uso de cateteres são as infecciosas, hemorrágicas e mecânicas.

Segundo o autor supracitado, a taxa de utilização de cateter para realização de HD é $25 \%$ superior ao de FAV, devido principalmente ao diagnóstico e consequentemente, referência tardia dos doentes renais aos serviços especializados, ocasionando a necessidade de acesso de urgência (ZICA, 2016).

Ressalta-se a importância de as intervenções de enfermagem abrangerem a valorização do autocuidado, teoria desenvolvida por Dorothea Elizabeth Orem em 1971, tornando o paciente participante de seu tratamento com o cuidado de si próprio, garantindo-Ihe mais autonomia e independência (SANTOS et al., 2011).

Desta maneira, a ação de enfermagem é fundamental para a prestação de cuidados com o cateter e também para estimular o doente renal crônico a realizar o autocuidado, conforme suas limitações e 
necessidades. Formulando um plano terapêutico individualizado e baseado na teoria do autocuidado, a fim de reduzir as possíveis complicações com o cateter e garantir maior qualidade de vida ao paciente. Destarte, o presente estudo tem como objetivo analisar o conhecimento de pacientes em hemodiálise sobre o autocuidado com o cateter venoso central.

\section{MÉTODO}

O estudo é descritivo com abordagem qualitativa, foi realizado no Serviço de Terapia Renal Substitutiva (STRS) da Fundação Pública Estadual Hospital de Clinicas Gaspar Vianna (FHCGV), no qual funciona em três turnos, nos dias de segunda a sábado e tendo o domingo para atendimentos emergenciais.

Foram incluídos na pesquisa pacientes matriculados no STRS, de ambos os sexos, submetidos a terapia do tipo HD por meio de cateter de curta ou longa permanência, maiores de 18 anos com capacidade de cognição/compreensão preservada para ser capaz de responder às questões da pesquisa por meio do diálogo coerente. Sendo excluídos os pacientes que possuíam FAV para a terapia e que, por qualquer motivo (cognitivo ou clínico), não eram responsáveis pelo próprio cuidado em relação ao cateter e portadores de necessidades especiais.

Após a aplicação dos critérios de inclusão e exclusão 11 pacientes foram convidados a participar da pesquisa. Dos quais, 02 se recusaram, reduzindo a amostra para 09 pacientes.

A pesquisa contou com um instrumento de coleta de dados, o Roteiro de Entrevista. Após a aprovação do Comitê de Ética em Pesquisa da FHCGV, sob o protocolo CAA:79697017.6.0000.0016, mediante o parecer $n^{\circ}$ 2.456.317, as coletas iniciaram em duas etapas, na primeira foi realizada a busca nos prontuários dos pacientes para a identificação dos dias e horários das sessões de hemodiálise, já a segunda etapa se deu com a realização das entrevistas com os pacientes que aceitaram compor o estudo.

A análise de dados seguiu o modelo de análise de conteúdo proposto por Bardin, o qual realiza a análise em três fases, a primeira é chamada de Pré-análise, onde foi criado o corpus do trabalho através da leitura das entrevistas. A segunda fase, de Exploração do material, no qual o corpus foi interpretado, e ocorreu classificação e categorização das informações. A última fase, denominada tratamento dos resultados, inferências e interpretação, foi realizada a interpretação dos dados conforme teoria escolhida (CÂMARA, 2013).

Em relação aos riscos e benefícios, a pesquisa apresentou riscos mínimos, pois foi utilizado o código alfanumérico com a letra "R", que representa Renal, seguida da sequência numérica garantindo o anonimato e quanto ao desconforto ou constrangimento no momento da entrevista, este foi minimizado pela garantia de local reservado para a coleta dos dados.

\section{RESULTADOS E DISCUSSÃO}

Da análise compreensiva emergiram 3 categorias: Conhecimento e significância da hemodiálise na vida dos pacientes renais crônicos; o autocuidado e o cotidiano do paciente renal crônico em hemodiálise e a autoestima e autoimagem do paciente renal crônico em hemodiálise.

\section{Conhecimento e significância da hemodiálise na vida dos pacientes renais crônicos}

Quando questionados sobre o que era hemodiálise os pacientes apresentaram respostas diferentes, dentre estas, obteve-se falas que demonstraram o nível de conhecimento sobre a hemodiálise e outras que evidenciaram o significado da hemodiálise em suas vidas. Conforme descrito abaixo:

"Hemodiálise é isso que eu to fazendo (pausa) não tenho certeza muito do que é hemodiálise, não entendo muito o que é, eu to fazendo hemodiálise por causa do rim (pausa) faço tem uns dois anos". (R1) 
É possível identificar uma insuficiência no conhecimento acerca do tratamento, mesmo quando o realizado a mais de um ano. Consoante Mendonça et al. (2013) em pesquisas realizadas com pacientes que realizavam HD com Cateter Duplo Lúmen (CDL) foi constatada como diagnóstico de enfermagem o conhecimento deficiente em $81 \%$ da amostra, reforçando a necessidade da intervenção da enfermagem para solucionar ou minimizar tal realidade por meio de educação em saúde, avaliando seu nível de compreensão e reforçando as informações sobre o tratamento e cuidado com o cateter.

Pode-se perceber que para a maioria dos pacientes a HD significa um tratamento e/ou esta relacionada ao rim, no entanto outras respostas foram muito expressivas quanto à significância da HD, sendo para alguns pacientes como algo bom e outros como ruim, como exposto:

"Hemodiálise para mim é sobrevivência!" (R5)

Outros entrevistados percebem a hemodiálise como vital, porém ruim, conforme demonstrado abaixo:

"Para mim é uma oportunidade para eu viver, porque sem ela a gente não vive, mesmo que seja estressante, a gente não quer falar com ninguém, as vezes fica 4 horas sentado[...]" (R7)

Para explicar a ambivalência de sentimento observado no que foi dito pelos participantes, Takemoto et al. (2011) considera a HD como responsável pela sobrevivência, diminuição de complicações e manutenção do bem-estar, ainda que restrinja à uma rotina cansativa, monótona e ocasione sofrimentos emocionais e psicológicos.

Chama-se atenção para a fala do participante R3 por ser dissonante dos demais quando se refere ao significado da HD, pode-se perceber na subjetividade que a HD é algo ruim:

"Hemodiálise para mim é uma doença." (R3)

Este sentimento é mencionado por Santos et al. (2011) ao descrever que o paciente em HD percebe a perda de sua autonomia, esperança e da saúde, visto que as doenças crônicas não têm cura e o paciente relaciona esse fato à possibilidade de diminuição do tempo de vida e condução a morte.

Diante das falas pode-se inferir que os pacientes demonstram forte vínculo emocional com a HD, dando-Ihe adjetivos e que o conhecimento sobre o tratamento não é visto como mais importante que os impactos que a HD causa na vida do paciente. No entanto, mesmo com as poucas respostas referentes ao conhecimento, pode-se perceber, que tal saber é mínimo, isso pode ocorrer devido as informações que não foram repassadas ao paciente ou este não conseguiu absorver devidamente o que lhes foi orientado, evidenciando fragilidade no sistema de educação em saúde.

Quando questionados a busca de informações sobre como cuidar de si, dentre os participantes, dois citaram a enfermagem em suas falas, um deles sentiu necessidade de obter mais informações e afirmou que as vezes não conseguia suprir tal demanda com os profissionais do setor. Necessitando realizar pesquisas por conta própria na internet, e a mesma afirmou que as informações encontradas geravam forte impacto emocional pela forma que são disponibilizadas. Conforme mostrado abaixo:

\section{"[...] a enfermagem falou que não podia molhar. Eles sempre falam" (R7)}

"Sempre sou muito questionadora, no hospital, lá em cima eu tenho amizade com os profissionais!.Na internet eu era 'meio coisa', porque a internet informa muito pesado, as vezes eu ficava para baixo. E em enfermagem é muito relativo. Muita coisa que eu sei a enfermagem não diz, eu que pesquiso." (R8)

Os pacientes R1, R3 afirmaram que não realizavam buscas por informações, dentre estes chama-se a atenção para a fala do paciente $\mathrm{R} 3$, o qual podemos inferir que manifestou interesse em recebe-las, pois demonstrou a curiosidade e vontade de aprendizado. Ressalta-se que esse paciente era idoso e com grau de escolaridade baixo, fatores que podem ter interferido na busca pelas informações através de meios eletrônicos.

\section{"[...] nunca procurei me informar não, não fico curioso." (R1)}


"não busco informações, mas se tiver tempo para explicar eu aceito." (R3)

Conforme Takemoto et al. (2011) não só a enfermagem, mas todos os profissionais da saúde precisam adequar suas falas de acordo com o grau de conhecimento dos pacientes, para garantir a comunicação efetiva e a promoção em saúde para prevenção de complicações no tratamento, pois como observado em sua pesquisa que $11,8 \%$ dos pacientes nunca estudaram e $29,4 \%$ possuía apenas o ensino fundamental incompleto.

$\mathrm{Na}$ fala do participante R5 percebe-se dissonância, pois, podemos inferir que em sua subjetividade, que a paciente possui a preocupação com o cuidado com sua mãe do que o autocuidado.

"[...] faço pergunta para o médico, nutrição o que tem comer, na internet eu busco mais informações para minha mãe que é diabética" (R5)

De acordo com Araujo et al. (2016)o profissional da saúde ao menosprezar os interesses do paciente renal crônico, acaba diminuindo o diálogo e não verificando as dúvidas e queixas, ocasionando prejuízos na assistência como negligencia e /ou abandono do tratamento.

\section{$O$ autocuidado e o cotidiano do paciente renal crônico em hemodiálise}

Quando questionados sobre o cuidado com o cateter durante o banho, na fala de oito participantes percebe-se que todos procuravam estratégias para não molhar o acesso, como a colocação de saco plástico e alguns pacientes quando possuíam condições financeiras compravam o curativo impermeável. Assim, podemos inferir que há a preocupação entre os participantes quanto a perda do acesso, pois para eles a manutenção do cateter é fundamental para a realização da hemodiálise e, portanto, para sua sobrevivência. Como pode ser verificado na fala de R3 e R7 :

\section{"[...] graças a Deus nunca molhei, coloco um plástico e põe um pano e evita molhar." (R3)}

"Eu boto um plástico, toda semana eu compro esparadrapo, as vezes compro o curativo impermeável, mas é caro." (R7)

O papel da enfermagem é significativo no repasse de orientações para este paciente exercer seu autocuidado, pois de acordo com National Kidney Foundation Kidney Disease Outcomes Quality - NKF KDOQI (2017), as orientações são parte do cuidado fundamental de enfermagem, onde o paciente que realiza hemodiálise, deverá ser informado sobre os cuidados com o acesso como, durante o banho para não molhar o cateter, a necessidade de cuidados para manter o curativo limpo e seco, reforçando que o mesmo só pode ser trocado e manipulado pela equipe de saúde responsável no serviço de seu tratamento.

Reforçando a prática da enfermagem, em sua teoria, Orem apresenta o conceito de autocuidado como sendo o indivíduo responsável por executar ações para a manutenção e qualidade de vida, de forma integra, desenvolvendo a habilidade de se engajar no autocuidado (ROCHA, 2010).

Durante as entrevistas, ao serem perguntados se tinham o costume de mexer no cateter e se realizavam o curativo em casa, os participantes de uma maneira geral deixam claro em suas falas que não mexem em seus acessos, pois foram informados que o curativo deve ser feito no momento em que realizam as sessões de hemodiálise, como referido nas falas de $R 8$ e R9, e destaca-se na fala do paciente R6 a subjetividade relacionada ao medo de perder o acesso de seu tratamento:

"Não mexo, o que eles me recomendam eu faço. Uma vez eu olhei assim...(olhou para o cateter), mas não lavo e nunca mexi." (R8)

"Não eu não posso mexer, só eles que mexem, eles que limpam quando venho fazer o curativo, só o enfermeiro." (R9)

"Eu mesmo não, sou medroso, não mexo em nada do hospital, eu não mexo, qualquer coisa tou avisando aí." (R6) 
Conforme Araújo et al. (2016) o ensino pode fazer diferença na condição de saúde do paciente, quando estes são bem informados e educados, geralmente se preocupam com a saúde e fazem o que é indispensável para mantê-la, pois o conhecimento ajuda a tomar decisões que os beneficiam em sua trajetória de tratamento.

Podemos inferir que estes pacientes, segundo as concepções de Dorothea Orem, se encontram no sistema parcialmente compensatório, ou seja, tanto a enfermeira quanto o paciente executam medidas ou ações para o autocuidado, onde o enfermeiro deverá reforçar as orientações para o autocuidado, para que estas façam parte dos hábitos de vida dos pacientes, gerando melhor qualidade de vida (SANTOS et al., 2011).

Buscou-se também informações quanto as atividades de lazer dos participantes e quais eram as medidas adotadas por eles para realizar o autocuidado com o cateter. Nas falas dos pacientes foi possível perceber que após o inicio do tratamento a vida social foi modificada. De maneira geral os participantes responderam que não costumavam sair para passear ou fazer alguma atividade para distração desde a descoberta da doença. Pode-se destacar nas falas de R3, R4 e R7:

"Depois que eu comecei a fazer o tratamento eu nunca mais sai." (R3)

"Antes eu gostava muito de sair quando eu não era doente, aí depois que eu adoeci eu não sai mais." (R4)

“Gostava de sair, mas agora não dá mais, fico na frente de casa...vou pra igreja." (R7)

Segundo Frazão et al. (2011) corroborando com as falas dos participantes a HD é uma terapia que modifica completamente a vida dos pacientes, pois além de ter que aceitar a sua condição de portador de uma doença crônica, que apesar de realizar tratamento, é irreversível, esta terapia muda a rotina e a vida de quem está submetido a ela. O tratamento dialítico resulta limitações no lazer e poderá causar tédio e a diminuição da qualidade de vida dos pacientes, muitas vezes abdicam ou tem seus prazeres, viagens e passeios restritos.

Ainda envolvendo o autocuidado com o cateter, os participantes ao serem perguntados se costumavam ir à praia ou piscina e de que maneira agiam com o seu acesso, estes responderam de forma geral em suas falas que se frequentassem estes locais eles não tomariam banho, pois não podem molhar o cateter. Sendo também, observado nas repostas que em decorrência de suas restrições, eles evitam realizar este tipo de lazer. Assim, podemos inferir que os pacientes reconhecem a importância de seu acesso central e por meio da subjetividade percebe-se os sentimentos como medo, ansiedade e insegurança envolvidos em suas falas.

"Não, não vou pra parte nenhuma, até queria ir um dia só olhar(pausa) olhar o pessoal tomar banho, por que se eu for pra uma praia eu só vou olhar o pessoal tomar banho, por que eu não posso molhar né, eu não posso cair na água. [...] faz muitos anos, faz muito tempo mesmo, nunca mais fui pra parte nenhuma, não posso molhar, na praia ia me jogar na água, aí ia molhar e ia me prejudicar. Sobre isso eu respeito muito [...] (R1)

Conforme Neves (2014), de maneira geral a DRC traz consequências, enfrentadas como negativas pelo paciente, em decorrência das restrições que a doença e o tratamento implicam e que podem atingir as necessidades de autoimagem e autoestima de forma negativa, o que pode interferir nas relações afetivas e seu convívio social. Por isso é importante que se mantenha o contato e convívio com amigos e familiares.

\section{A autoestima e autoimagem do paciente renal crônico em hemodiálise}

A rotina do paciente renal é modificada em função das limitações impostas pelo tratamento. Enfrentará mudanças que incluem restrições alimentares, dificuldades para manter-se empregado, diminuição das atividades sociais, limitação da expectativa de vida, demostrando depressão e medo da morte por ser renal crônico. E, por fim, a perda da autoestima e alteração da autoimagem (NEVES, 2014). 
Em relação a esses fatores, quando os participantes da pesquisa foram questionados se sentiam vergonha do cateter, percebeu-se que nas falas dos pacientes R2 e R3 que não houve qualquer problema com relação à presença do acesso:

"não, antes porque foi assim, quando eu entrei no Barros de eu tive acompanhamento psicológico, então a doutora que eu sempre falava com ela lá, ela chegou a fazer essa pergunta pra mim, e eu nunca tive vergonha não[...]" (R2)

"não, não tenho não." (R3)

Já os pacientes R1 e R5, ainda que tenham relatado não sentir vergonha da presença do acesso, pode-se inferir que os mesmos revelam uma insatisfação com o posicionamento do cateter por ser um local visível e já tentaram esconder ou até mesmo disfarçar o cateter, como pode ser visto nas falas abaixo:

"Não, não tinha vergonha, mas era bocado feio né, as vezes no pescoço era feio, mas não podia fazer nada. As vezes tinha um ai que "sentava" bem e eu cobria, sentava e com a camisa cobria, aí não aparecia, as vezes uns ficam em "pé", aí quando vem perfeito ele ficava deitado e eu vestia a camisa e sumia." (R1)

"Não porque não aparece, não dá para vê aí uso roupa normal e dá para esconder, dobro a pontinha!" (R5)

O participante R9 afirmou não sentir vergonha quando questionado, porém, em outro momento da entrevista, quando foi indagado acerca de sua eventual frequência em praias ou clubes, o mesmo afirmou que não costuma ir a tais lugares, uma vez que teria de proteger o acesso de alguma forma, fato que geraria constrangimentos. Podemos inferir que o paciente defronta com diversos sentimentos estressantes, como raiva, negação, vergonha, incerteza. Pois, tem uma doença que veio para ficar e, além disso, traz consigo um tratamento inevitável. Segue abaixo a fala do mesmo:

"[...] acho que ia ter que meter no saco também, né? O que eu vou fazer na praia para passar vergonha?" (R9)

Em relação à autoimagem Araujo et al. (2016) afirma que uma das complicações geradas nos pacientes em diálise, decorrentes da autoimagem prejudicada, é a depressão, podendo existir também outros transtornos psicológicos associados.

Ainda se tratando do sentimento de vergonha, os participantes R6, R7 e R8 afirmaram ter vergonha do cateter, e tentavam escondê-lo com suas roupas, conforme descrito nas falas abaixo:

"Ah sempre né, a gente coloca uma roupa e fica logo preocupada se vai aparecer, eu uso roupa que cobre e dobro um pouco para não a aparecer as vezes." (R6)

"Quando tava no braço eu escondia, o povo é muito curioso, eu escondia na camisa, eles querem saber o que é.” (R7)

"Com fístula não queria sair porque ficava muito preocupada porque esteticamente é horrível e no pescoço tinha vergonha." (R8)

Gonzalez et al. (2017) afirmam que o cateter afeta o paciente renal crônico em vários âmbitos de sua rotina e o mesmo busca formas de se adaptar à nova realidade em situações como vida sexual, lazer, higiene, banho e outras. Reiterando assim, o papel da enfermagem no desenvolvimento de atividades de ensino ou práticas educativas de saúde visando a prevenção de complicações através do autocuidado, possibilitando melhor adaptação do mesmo. Conscientizando-o da importância da mudança de comportamentos e atitudes a fim de conquistar autoestima, vontade de aprender, o cuidado com o cateter, proporcionando uma convivência mais feliz no seio familiar e no contexto social.

Este estudo verificou-se carência de conhecimento sobre o autocuidado com o cateter venoso central para hemodiálise, que independia do tempo de tratamento, tal carência pode ocorrer tanto pela falta de repasse 
de informações a estes pacientes, bem como pela ausência ou diminuição da absorção das informações repassadas, evidenciando fragilidades no processo de educação em saúde dos profissionais para com esses pacientes. Necessitando assim de estratégias para intensificação de ações educativas analisando as necessidades especificas, dificuldades e limitações dos pacientes nesse processo. A prática do autocuidado necessita ser estimulada pela equipe de enfermagem através do apoio e do fornecimento de orientações, uma vez que se destina a melhorar a qualidade de vida dos pacientes renais crônicos que realizam hemodiálise.

\section{CONCLUSÃO}

O paciente necessita ter conhecimento sobre a doença, sintomas e limitações físicas, bem como os cuidados recomendados com cateter venoso central e ter adesão ao tratamento. Percebemos com esse estudo a grande relevância do tema para obtenção de conhecimento para a comunidade científica que poderá utiliza-lo para posteriores pesquisas e para o setor no qual foi desenvolvida o estudo já que as pesquisadoras ofertaram o feedback do resultado da pesquisa, motivando a construção de tecnologias educativas para uso com estes pacientes, além da implementação da Sistematização da Assistência de Enfermagem, a qual poderá identificar as deficiências no autocuidado e implementar as intervenções de enfermagem minimizando tal problemática.

\section{REFERENCIAS}

1. ARAÚJO JB, SOUZA NETO VL, ANJOSEU et al. Cotidiano de pacientes renais crônicos submetidos à hemodialise: expectativas, modificações e relações sociais.J. Res.: fundam. care. Online,2016; 8(4): 996-5001.

2. BASTOS MG, KIRSZTAJN GM. Doença renal crônica: importância do diagnóstico precoce, encaminhamento imediato e abordagem interdisciplinar estruturada para melhora do desfecho em pacientes ainda não submetidos à diálise. Jornal Brasileiro de Nefrologia, 2011; 33(1):93-108.

3. CÂMARA RH. Análise de conteúdo: da teoria à prática em pesquisas sociais aplicadas às organizações. Revista Interinstitucional de Psicologia, 2013; 6(2): 179-191.

4. FRAZÃO CMFQ, RAMOS VP, LIRA ALBC et al.Qualidade de vida de pacientes submetidos à hemodiálise. Rev. enferm. UERJ. Rio de Janeiro,2011;19(4) :577-82.

5. GONZALEZ CM, TEIXEIRAMLO, BRANCO EMSC et al. Cuidado educativo compartido: estrategia de acción delaenfermería junto a usuários coninsuficiencia renal crónica. Rev. baiana enferm, 2017; 31(3): 17536.

6. GUIMARÃES GL, GOVEIAVR, QUISPE MENDONZA IY et al. Intervenções de enfermagem no paciente em hemodiálise por cateter venoso central. Revista enfermagem UFPEonline, 2017; 11(3): 1127-35.

7. MENDONÇA NN, DUTRA MG, FUNGHETTO SSet al. Diagnósticos de enfermagem de pacientes hemodialíticos em uso do cateter duplo lúmen. R. Enferm. Cent. O. Min. Minas Gerais,2013; 3(2): 632-644.

8. NATIONAL KIDNEY FOUNDATION-DIALYSIS OUTCOMES QUALITY INITIATIVE (KDOQI). Clinical practice guidelines for vascular access. New York: 2017.

9. NEVES CN.Compartilhamento de Saberes e Práticas da Equipe de Enfermagem Sobre Cuidados com a Pele na Doença Renal Crônica: Uma Estratégia Educativa. Dissertação (mestrado) - Escola de Enfermagem Ana Nery. Universidade Federal do Rio de Janeiro. Rio de Janeiro, 2014.

10. ROCHA RPF. Necessidade de orientação de enfermagem para o autocuidado visando a qualidade de vida de clientes em terapia de hemodiálise. Dissertação (Mestrado) - Universidade Estadual do Rio de Janeiro. Rio de Janeiro, 2010.

11. SALIMENA AMO, SOUZAMO, MELOMCSC et al. O cotidiano da mulher em hemodiálise. Rev. Cuidado é fundamental, 2016; 8(3):4636-4643.

12. SANTOS I, ROCHA RPF, BERARDINELLI LMM et al. Qualidade de vida de clientes em hemodiálise e necessidades de orientação de enfermagem para o autocuidado, 2011; 15(1): 3-38.

13. SOCIEDADE BRASILEIRA DE NEFROLOGIA. SBN Informa. 2016.

14. TAKEMOTO AY, OKUBOP, BEDENDOJet al. Avaliação da qualidade de vida em idosos submetidos ao tratamento hemodialítico. Rev. Gaúcha Enferm. Porto Alegre, 2011; 32(2): 256-62.

15. ZICA DS. Manual educativo sobre cuidados com acesso vascular para hemodiálise.Dissertação (Mestrado Profissional em Ciências Aplicadas à Saúde) - Universidade do Vale do Sapucaí, Porto Alegre. 2016. 